\title{
Mode-Locking Matter with Light
}

\author{
Bern Kohler, Jeffrey L. Krause," Ferenc Raksi, Christoph Rose-Petruck, Robert M. Whitnell, \\ Kent R. Wilson, Vladislav V. Yakovlev, and YiJing Yan ${ }^{\dagger}$
}

Department of Chemistry, University of California, San Diego, La Jolla, California 92093-0339

\author{
Shaul Mukamel \\ Department of Chemistry, University of Rochester, Rochester, New York 14627 \\ Received: May 18, 1993; In Final Form: August 5, $1993^{\circ}$
}

\begin{abstract}
Theory and experiments are currently being developed in our laboratory to use tailored light fields to control the dynamics of molecular reactions. We have recently shown theoretically that a properly chirped light pulse can be used to overcome the natural tendency of wave packets to spread on anharmonic molecular potential energy surfaces. The optimal chirp rate can be readily obtained by solving a linear optimal control equation. In this paper we analyze a number of aspects crucial to the success of the experiments. We test the robustness of the optimal electric field with respect to variations in the field parameters (chirp rates, pulse duration, and carrier frequency). We also study the effects of finite temperature by using a thermal vibrational distribution as the initial state in the dynamics. Finally, we describe the experimental apparatus that we plan to use to perform the control experiments, and discuss several technical issues related to synthesizing the optimal field and detecting the focused wave packet.
\end{abstract}

\section{Introduction}

In previous papers 1,2 we have presented a formalism for the weak field control of molecular dynamics and computational examples of the implementation of this theory. The theory enables us to predict the light field which best achieves a given objective. Using realistic potentials for the $\mathrm{I}_{2}$ and $\mathrm{Na}_{2}$ molecules, we showed ${ }^{2}$ that this globally optimal field can be used to control the evolution of a vibrational wave packet on an excited electronic potential energy surface. The ability to steer a wave packet to a desired region of configuration or phase (position/momentum) space at a selected final time by mode-locking the vibrational states of a molecule is a first step in controlling the outcome of a chemical reaction. For reviews of alternative approaches to this problem, see refs $3-7$.

The globally optimal field can be well approximated by a simple functional form consisting of a series of Gaussian pulses with time dependent phase functions containing phase constants, center frequencies, and linear and quadratic chirps. Although the Gaussians do not reproduce the optimal field perfectly, they are still quite successful at driving the molecular system toward the desired goal. Furthermore, the parameters of the best fit fields are experimentally reasonable, thereby making the synthesis of such pulses feasible.

In this paper, we concentrate on a number of aspects crucial to observing in the laboratory the effects that we have predicted. One concerns the robustness of our results with respect to small changes in the parameters of the optimal field. This is important because neither the theory nor the experiments are perfect, and if the region of stability in parameter space is small, we may never reach it in the experiments or locate it reliably in the calculations.

In our previous calculations ${ }^{2}$ we neglected the effects of finite temperature on the dynamics. While we have previously treated thermal effects analytically and presented computational results for displaced harmonic oscillators, ${ }^{1}$ our initial work on the molecular examples assumed a pure $\nu^{\prime \prime}=0$ initial state, which corresponds to a "temperature" of $0 \mathrm{~K}$. In this paper, we analyze

+ Also at the Institute for Nonlinear Science, University of California, San Diego

- Abstract published in Advance ACS Abstracts, November 1, 1993. the effects of the thermal vibrational distribution on the ground electronic state by performing the calculations with a mixed state consisting of a Boltzmann weighted sum of vibrational levels.

Finally, we are constructing experiments ${ }^{8}$ to control molecular dynamics using our calculations as a guide to the experimental design. We describe the apparatus we are building to synthesize the optimal fields and to measure the resulting quantum control and discuss some of its characteristics.

\section{Theory}

In this section we review the relevant quantum control equations. We refer the reader to refs 1 and 2 for a more detailed derivation. For related work by other authors, see refs 9-14.

We assume that two electronic potential energy surfaces, a ground state and an excited state, are the only states relevant to the dynamics. We further specialize to the case of a diatomic molecule and consider only its vibrational coordinate. The theory proceeds by choosing a target, i.e., the objective of the control, and then calculating the optimal field that excites a wave packet which has the maximal overlap with the target at a selected final time, $t_{f}$. The initial state is then propagated in the presence of the optimal field to determine how well the target is attained.

We note that the theory is derived in the weak field limit (we treat the more general strong field case elsewherel). However, this limit does not imply the limit of zero electric field but refers to the regime in which the molecular response is linear in the field strength. In this respect, the weak field limit can actually extend to quite high intensities. In fact, most spectroscopic observations to date have been performed in this limit.

We begin with a conventional wave packet propagation in the absence of the field. The time-propagated wave packet on the excited state can be written as

$$
\left|\psi_{\mathrm{e}}^{0}(t)\right\rangle=\mathrm{e}^{-i\left(H_{\mathrm{e}}-\epsilon_{\mathrm{r}^{\prime}}\right) t / \hbar}\left|\psi_{\mathrm{e}}^{0}(0)\right\rangle
$$

In this equation $H_{\mathrm{e}}$ is the excited-state Hamiltonian, and the initial state $\left|\psi_{e}^{0}(0)\right\rangle$ is

$$
\left|\psi_{\mathrm{e}}^{0}(0)\right\rangle=\mu\left|\nu^{\prime \prime}\right\rangle
$$

where $\left|\nu^{\prime \prime}\right\rangle$ is a vibrational level on the ground electronic state with eigenenergy $\epsilon_{v^{\prime \prime}}$. For simplicity we assume in the following 
that the dipole moment $\mu$ is a constant (Condon approximation). The time propagation is performed with the Chebychev method, ${ }^{15}$ and the Hamiltonian is discretized using the discrete variable representation (DVR). ${ }^{16,17}$ The time-evolving wave packet is stored at each time step so that the (expensive) propagation need only be performed once.

The kernel of the control problem ${ }^{1,2}$ is the molecular response function $M$, which can be constructed in the weak field limit as a convolution of the time-evolving wave packet with a target operator $\hat{A}$.

$$
M\left(t_{2}, t_{1}\right)=\left\langle\psi_{\mathrm{e}}^{0}\left(t_{2}\right)|\hat{A}| \psi_{\mathrm{e}}^{0}\left(t_{2}+t_{1}\right)\right\rangle
$$

Notice that $M$ depends only on properties of the material and the target and has no dependence on the electric field. Thus $\left|\psi_{\mathrm{e}}^{0}(t)\right\rangle$ can be stored and then recycled to study additional targets. In this and previous papers, we choose the target to be a minimum uncertainty Gaussian distribution centered at a particular configuration $\bar{x}$ and momentum $\bar{p}$ at a final time $t_{f}$. Thus the target operator, $\hat{A}=\left|\Phi_{A}\right\rangle\left\langle\Phi_{A}\right|$, is a projection operator onto the target wave function $\Phi_{A}(x),{ }^{18,19}$

$$
\Phi_{A}(x)=\left(2 \pi w_{q q}\right)^{-1 / 4} \exp \left[-\frac{1}{4 w_{q q}}(x-\bar{x})^{2}+\frac{\mathrm{i} \tilde{p}}{\hbar}(x-\bar{x})\right]
$$

where $w_{q q}$ is the variance of the target in position $\left(=(\Delta x)^{2}\right)$. This equation also implies that $w_{p p} w_{q q}=\hbar^{2} / 4$, where $w_{p p}$ is the variance of the target in momentum $\left(=(\Delta p)^{2}\right) . \Phi_{A}(x)$ is therefore a minimum uncertainty wave packet; that is, $\Delta x \Delta p=h / 2$. The objective of the control problem is to find the electric field which excites a vibrational wave packet that best overlaps with the target at $t_{f}$. A related procedure has been suggested by Averbukh and Shapiro ${ }^{20}$ to determine the electric field that produces an optimally squeezed wave packet and demonstrated for a system of displaced harmonic oscillators. We emphasize that eq 4 is only one possible choice of target and that any desired target can be expressed in terms of an appropriate target operator and phase-space distribution. In a polyatomic case, for example, the target operator might be a projection onto a particular product arrangement. The target can also be an optimal intermediate wave packet which is then driven to a final target by a subsequent interaction with another optimal light field. Thus, by interacting with one light field to focus in phase space, and a second field to carry the molecule to a desired final state, the products of a reaction can, in principle, be controlled.

We next proceed to find the optimal field by diagonalizing $M^{\mathrm{s}}$, a symmetrized version of the $M$ function,

$$
\int_{0}^{t_{f}} \mathrm{~d} \tau^{\prime} M^{S}\left(\tau, \tau^{\prime}\right) E\left(\tau^{\prime}\right)=\lambda \mathrm{E}(\tau)
$$

where

$$
M^{S}\left(\tau, \tau^{\prime}\right)=\left[M^{S}\left(\tau^{\prime}, \tau\right)\right]^{*}=M\left(t_{f}-\tau, \tau-\tau^{\prime}\right)
$$

for $\tau^{\prime} \leq \tau$. By discretization of $M^{S}$ and $E$ on a grid of $\tau$ and $\tau^{\prime}$, eq 5 can be recast as a conventional matrix eigenvalue equation,

$$
\mathbf{M}^{\mathbf{S}} \mathbf{E}=\lambda \mathbf{E}
$$

In eqs 5 and 7 , the eigenvalues $\lambda$ are the yields with respect to the incident field energy, and the eigenvectors associated with $\lambda$ are the optimal fields. The eigenvector associated with the largest eigenvalue is therefore the globally optimal field, in the sense that it is the weak field which excites a wave packet that has the best possible overlap with the target at the chosen final time. This expression illustrates one advantage of operating in the weak field limit. The globally optimal field is obtained directly from the solution of an eigenequation. In the strong field case, control equations can be derived, ${ }^{1,4,9-13}$ but they must be solved iteratively, rather than directly, which can involve repeating the wave packet propagation many times. In addition, the iterative procedure is not guaranteed to converge to the globally optimal field but may instead converge to a local extremum, especially in the (general) situation of many local minima with overlapping basins of attraction. However, strong field effects are intensely interesting, and we believe that both weak and strong field solutions are worth pursuing for computational as well as experimental reasons.

Having obtained the globally optimal field, we next calculate the effect of that field on the system, which can be written in the weak field limit as

$$
\psi_{\mathrm{e}}(t)=\hbar^{-1} \int_{0}^{t} \mathrm{~d} \tau E(\tau) \psi_{\mathrm{e}}^{0}(t-\tau)
$$

Once again, the $\psi_{e}^{0}(t)$ quantities calculated previously can be re-used for this convolution.

To determine how well we have attained our goal, we define an achievement function ${ }^{2}$

$$
\alpha(t)=\frac{\left|\left\langle\Phi_{A} \mid \psi_{\mathrm{e}}(t)\right\rangle\right|}{\left\langle\Phi_{A} \mid \Phi_{A}\right\rangle^{1 / 2}\left\langle\psi_{\mathrm{e}}\left(t_{\mathrm{f}}\right) \mid \psi_{\mathrm{e}}\left(t_{\mathrm{f}}\right)\right\rangle^{1 / 2}}
$$

The achievement $\alpha$ is normalized at the final time $t_{\mathrm{f}}$ and is a dimensionless quantity with values between zero and one that can be used for an arbitrary weak control field. It differs from the yield, $\lambda$, in that $\lambda$ is valid only for the optimal weak field and is scaled by the field strength, which is arbitrary in the weak field limit.

The procedure outlined above gives the globally optimal weak field for a particular material system and choice of target. However, in an experiment, only a restricted universe of light fields can be synthesized. We have chosen as an illustration to fit the globally optimal field by one possible functional form that can, in principle, be constructed in the laboratory. ${ }^{5,21}$ The representation of this complex field is a series of Gaussians of the form

$$
E(t)=\sum_{j} E_{j}(t)
$$

where

$$
E_{j}(t)=A_{j} \mathrm{e}^{-1 / 2\left(t-t_{j}\right)^{2} / \Gamma_{j}^{2}} \mathrm{e}^{-i \varphi_{j}(t)} \mathrm{e}^{-i \omega_{e j}\left(t-t_{j}\right)}
$$

$A_{j}$ is the amplitude of the $j$ th pulse, $l_{j}$ is the center time of the $j$ th pulse, and $\omega_{e g}$ is the difference in energy between the minima of the excited-and ground-state potentials. The temporal width of the pulse $(\mathrm{fwhm})$ is $(\ln 16)^{1 / 2} \Gamma_{j}$. The time-dependent phase $\varphi_{j}(\mathrm{t})$ can be expanded in a Taylor series containing a phase constant $\bar{\varphi}_{j}$, center frequency $\bar{\omega}_{j}$, as well as linear and quadratic chirps $c_{j}^{\prime}$ and $c^{\prime \prime}$, respectively,

$$
\varphi_{j}(t)=\bar{\varphi}_{j}+\bar{\omega}_{j}\left(t-\bar{l}_{j}\right)+{ }^{1} /{ }_{2} c_{\mathrm{j}}^{\prime}\left(t-\boldsymbol{l}_{j}\right)^{2}+1 /{ }_{6} c^{\prime \prime}{ }_{\mathrm{j}}\left(t-\bar{I}_{j}\right)^{3}
$$

For a field consisting of a single pulse, $\bar{\varphi}$ is arbitrary and irrelevant. We use a conventional nonlinear fitting routine 22 to find the best fit values of the parameters. Note that this procedure gives the best fit of the optimal field by a particular parameterized functional form. It does not, in general, guarantee that the parameters so obtained are those which optimize the achievement, $\alpha(t)$. We have used an alternative method which uses a conjugate gradient method to maximize the achievement with respect to the parameters ${ }^{2}$ but will not discuss the results of that optimization procedure further here.

\section{Robustness}

An important criterion for the success of control experiments, such as those under construction in our laboratory, is for the optimal fields predicted by the theory to be robust with respect to variations in the parameters. Such variations can be caused by inaccuracies in the theoretical model or the material description (Hamiltonian) which is its input, or experimental uncertainties, including the inability to create fields that exactly match the 


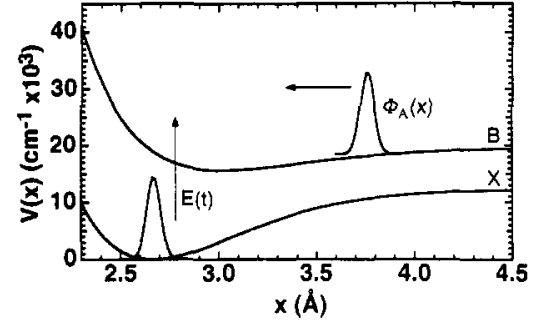

Figure 1. Potential energy curves for the $I_{2}$ ground $X$ and excited $B$ states. The target for the molecular reflectron is a minimum uncertainty wave packet with an incoming momentum (momentum to the left) in the bound region of the potential surface. The objective is to find the optimal field $E(t)$ that creates a time-evolving wave packet on the excited surface which best overlaps the target at a given final time $t_{f}$.
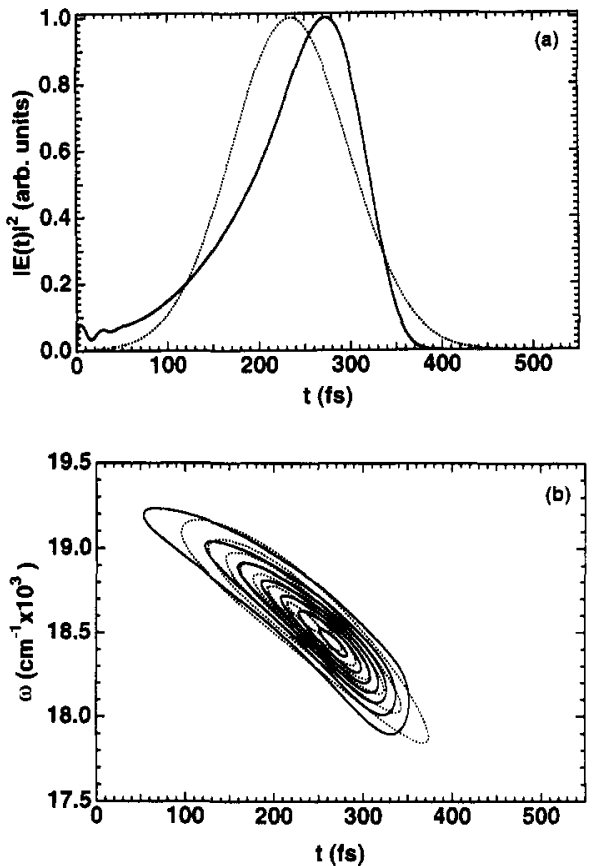

Figure 2. Globally optimal and Gaussian best fit fields for the reflectron at $T=0 \mathrm{~K}$. (a) Intensity $|E(t)|^{2}$ for the optimal field (solid line) and best fit field (dotted line). The intensity units are arbitrary, as long as the weak field limit is valid. (b) Wigner representation for the globally optimal field (solid contours) and best fit field (dotted contours)

specifications of the theoretical optimal field ${ }^{8}$ To test the robustness of our results, we analyze in this section one of the cases we have presented previously, the $\mathrm{I}_{2}$ reflectron, ${ }^{2}$ and discuss the effects of varying the parameters of the field.

For the reflectron (see Figure 1), our chosen goal is to create a minimum uncertainty wave packet at a final time of 550 fs on the excited B state of $I_{2}$ centered at a position $\bar{x}=3.72 \AA$ with an incoming center momentum (momentum to the left in Figure 1) corresponding to a kinetic energy $p^{2} / 2 m=0.05 \mathrm{eV}$, where $m$ is the $\mathrm{I}_{2}$ reduced mass. The energy of the target $\left\langle\Phi_{A}|H| \Phi_{A}\right\rangle$ relative to the minimum of the B state potential is $2867 \mathrm{~cm}^{-1}$, placing it in the bound region of the spectrum, $1853 \mathrm{~cm}^{-1}$ below dissociation. The shift of the bottom of the B state relative to the bottom of the ground X state, $\omega_{\mathrm{eg}}$, is $15769 \mathrm{~cm}^{-1}$. The $I_{2}$ potentials used in this work are as presented previously. ${ }^{2}$ In Figure 2 we show the globally optimal electric field for the reflectron and the Gaussian function of eqs $10-12$ that best fits the optimal field. The parameters of the best fit field are presented in Table I. Figure $2 a$ shows $|E(t)|^{2}$ for the optimal and best fit fields, and Figure $2 b$ shows a modified Wigner representation,

$$
F_{w}(t, \omega)=2 \operatorname{Re} \int_{0}^{\infty} \mathrm{d} \tau \mathrm{e}^{-i \omega \tau} E^{*}(t+\tau / 2) E(t-\tau / 2) \mathrm{e}^{-\tau^{2} / \tau_{c}^{2}}
$$

of the optimal and best fit fields. In eq 13, the self-interference
TABLE I: Best Fit Parameters for the Reflectron

\begin{tabular}{clcc}
\hline parameter & \multicolumn{1}{c}{ best fit value } & parameter & \multicolumn{1}{c}{ best fit value } \\
\hline$A$ & 1.0 & $\bar{\omega}+\omega_{\mathrm{eg}}$ & $18580 \mathrm{~cm}^{-1}$ \\
$\bar{l}$ & $234 \mathrm{fs}$ & $c^{\prime}$ & $-4.3 \mathrm{~cm}^{-1} / \mathrm{fs}$ \\
$\Gamma$ & $90 \mathrm{fs}(151-\mathrm{fs} \mathrm{fwhm})$ & $c^{\prime \prime}$ & $-1.1 \times 10^{-2} \mathrm{~cm}^{-1} / \mathrm{fs}^{2}$ \\
$\bar{\varphi}$ & - & &
\end{tabular}

TABLE II: Reflectron Achievements as a Function of Varying the Parameters of the Best Fit Field

\begin{tabular}{lclc}
\hline \multicolumn{1}{c}{ case } & achievement & \multicolumn{1}{c}{ case } & achievement \\
\hline globally optimal field & 0.97 & $c^{\prime} \rightarrow c^{\prime} \times 2 ; c^{\prime \prime} \rightarrow 0$ & 0.64 \\
best Gaussian fit & 0.94 & $c^{\prime} \rightarrow-c^{\prime} ; c^{\prime \prime} \rightarrow 0$ & 0.34 \\
$c^{\prime \prime} \rightarrow 0$ & 0.89 & $\Gamma \rightarrow \Gamma \times 2$ & 0.91 \\
$c^{\prime \prime} \rightarrow-c^{\prime \prime}$ & 0.84 & $\Gamma \rightarrow \Gamma / 2$ & 0.82 \\
$c^{\prime} \rightarrow 0 ; c^{\prime \prime} \rightarrow 0$ & 0.46 & $\bar{\omega} \rightarrow \bar{\omega}+50 \mathrm{~cm}^{-1}$ & 0.77 \\
$c^{\prime} \rightarrow c^{\prime} \times 2 ; c^{\prime \prime} \rightarrow 0$ & 0.54 & $\bar{\omega} \rightarrow \bar{\omega}-50 \mathrm{~cm}^{-1}$ & 0.79
\end{tabular}

of the field present in the standard Wigner representation has been filtered out with a Gaussian window function. The parameter $\tau_{\mathrm{c}}$ is chosen to be on the order of the temporal duration of the pulse, and therefore its effect on the spectral bandwidth is minimal. The Gaussian filter has no effect on the temporal bandwidth.

Several points are evident from Figure 2 and Table I. First, the optimal and best fit fields display a sizable negative chirp. Thus, the high-energy components of the wave packet are excited before the low-energy components. It is the presence of this chirp that causes the focusing of the wave packet at $t_{\mathrm{f}}$. The reflectron operates by creating a wave packet in which the vibrational modes are phase-locked. This packet reflects off the soft outer wall of the potential in such a way that a narrow wave packet is produced at the selected final time. The high-energy components travel "further" than the low-energy components, to more anharmonic regions of the potential, where they feel less force returning them to the target region. Since their traversal time is longer, they must be created first. At $t_{f}$, the high-energy components "catch up" with the low-energy components and interfere constructively to form a narrow wave packet that has maximal overlap with the target.

Another point evident from Figure 2 and Table $I$ is that the optimal field is quite simple, and the parameters of the best fit field are reasonable, given current laser technology. The spectral bandwidth (fwhm) corresponds to a transform limited pulse of 21 fs. The carrier frequency of $18580 \mathrm{~cm}^{-1}$, or $538 \mathrm{~nm}$, corresponds to a vibronic excitation energy of $2921 \mathrm{~cm}^{-1}$ above the zero of the B state, which is nearly, but not exactly, equal to the target energy of $2867 \mathrm{~cm}^{-1}$. The linear chirp of $-4.3 \mathrm{~cm}^{-1} / \mathrm{fs}$ is of a magnitude that can be created by standard optical elements (gratings, prisms, etc.). Finally, we note that the achievement for the globally optimal field is 0.97 , while the achievement for the Gaussian best fit field is 0.94 . As can be seen in Figure 2, the best fit field is not a perfect match to the optimal field. In particular, the Gaussian form cannot reproduce the asymmetry in the optimal field, yet it still achieves $97 \%$ of the optimal achievement. This is one indication that the optimal field is in fact quite robust. In what follows we investigate in more detail the effect of variations in the parameters of the field on the achievement. We show the results of several such tests in Table II.

As can be seen in Table I, and by the curvature of the Wigner representations in Figure 2, the optimal and best fit fields contain a quadratic chirp. While both linear and quadratic chirps are fairly easy to make in the laboratory, they cannot be controlled with absolute precision. It may also be difficult to control them independently. Fortunately, as can be seen in Table II, the effect of the quadratic chirp on the achievement is not dramatic. Eliminating it completely causes the achievement to decrease to $92 \%$ of the optimal, and even changing its sign causes a decrease to only $89 \%$ of the optimal.

As might be expected from the classical description of the mechanism of the reflectron described above, the magnitude and 
sign of the linear chirp are quite important to the dynamics. Using a transform limited pulse, or a pulse in which the quadratic and linear chirps have been set to zero, we obtain an achievement of $47 \%$ of the optimal. Using a value of twice the optimal linear chirp gives an achievement of $56 \%$ of the optimal, while half the optimal chirp gives $66 \%$ of the optimal. However, changing the sign of the chirp reduces the achievement to $35 \%$ of the optimal. In designing an experiment, such as the pump-probe or pumpdump experiments described below, focusing a wave packet at a location that has maximum Franck-Condon overlap with the final state should increase the measured signal dramatically. Our prediction, then, is that the results will be quite sensitive to the linear chirp. Nonetheless, the variations in the linear chirp in Table II are substantial, and yet considerable control remains. This again is an indication that our results are robust to small fluctuations in the parameters. We also note that the achievements listed in Table II for the cases in which we have varied the best fit parameters are lower limits to the achievements attainable with respect to the remaining parameters. That is, we did not change the linear chirp, for example, and then re-optimize the remaining parameters. Doing so would undoubtedly increase the achievement.

The effects of altering the temporal duration of the pulse are not as dramatic as changing the chirp. Using twice the optimal temporal duration $\Gamma$ results in $94 \%$ of the optimal achievement, while a pulse of half the optimal duration yields an achievement of $85 \%$ of the optimal. The result of changing the temporal duration of the pulse while leaving the chirps fixed is to focus the wave packet before the target (if $\Gamma$ is greater than the optimal value) or after the target (if $\Gamma$ is less than the optimal). This robustness with respect to the temporal duration is encouraging, because one of the more difficult aspects of the reflectron experiment, as discussed below, is to create a pulse with sufficient bandwidth and intensity at the desired frequency. The spectral bandwidth required to synthesize the reflectron pulse is not simply the inverse of the temporal fwhm of the optimal field but is instead the bandwidth of the transform limited pulse that is the input to the device that makes the chirps. Thus, for the reflectron, the temporal duration of the required input pulse is not 151 -fs fwhm as might be assumed from Table $I$, but rather $21 \mathrm{fs}$, which is the duration required to support the spectral bandwidth. Intense pulses of such duration are difficult, but not impossible, to make.

Finally, the effect of changing the frequency shift of the optimal field is fairly large. A change of $+50 \mathrm{~cm}^{-1}$ diminishes the achievement to $79 \%$ of the optimal,and $-50 \mathrm{~cm}^{-1}$, to $81 \%$ of the optimal. The reflectron depends upon components of the wave packet with different frequencies and therefore different transit times interfering constructively in the target region. Detuning the carrier frequency of the dump pulse causes excitation of more or less high-or low-energy components and spoils the constructive interference. However, $50 \mathrm{~cm}^{-1}$ is a rather large frequency shift and provided that light of the desired frequency can be made (via nonlinear frequency mixing, white light generation, etc.), frequency control of much better accuracy than this should be attainable.

\section{Finite Temperature}

One aspect of the dynamics not treated in our previous studies of the reflectron ${ }^{2}$ is the effect of finite temperature. That is, we assumed that $\mathrm{I}_{2}$ begins in a pure $\nu^{\prime \prime}=0$ ground state, corresponding to a temperature of $T=0 \mathrm{~K}$. In reality, the vibrational levels of the ground electronic state are usually populated with a Boltzmann distribution. To include the effects of finite temperature, we must calculate the effect of the optimal field on a mixed state rather than a pure state. In this case, the theory is better adapted to the density matrix formalism in which it was originally derived, ${ }^{1}$ rather than the Hilbert space formalism and Schrödinger representation presented in section II.
In the linear or weak field regime, the molecular response function for a mixed state is simply the weighted sum of the $M$ functions for the vibrational states that have significant population

$$
M=\sum_{v^{\prime \prime}} W_{\nu^{\prime \prime}} M_{v^{\prime \prime}}
$$

where $W_{\nu^{\prime \prime}}$ is the Boltzmann factor

$$
W_{\nu^{\prime \prime}}=\mathrm{e}^{-\epsilon_{\nu^{\prime}} / k T} / \sum_{\nu^{\prime \prime}} \mathrm{e}^{-\epsilon_{\nu^{\prime \prime}} / k T}
$$

$k$ is the Boltzmann constant and $T$ is the temperature. The $M_{\nu^{\prime \prime}}$ functions are calculated as in eqs $1-3$, with initial states $\left|\nu^{\prime \prime}\right\rangle$ representing the various $\nu^{\prime \prime}$ vibrational levels of the ground state. The $M$ function is then symmetrized and diagonalized as in eqs 5 and 6 . To calculate the achievement, we use a density matrix formalism ${ }^{1,2}$ in which

$$
\alpha(t)=\left\{\frac{\operatorname{Tr}[\hat{A} \rho(t)]}{\operatorname{Tr}[\hat{A}] \operatorname{Tr}\left[\rho\left(t_{\mathrm{f}}\right)\right]}\right\}^{1 / 2}=\left\{\frac{\sum_{\nu^{\prime \prime}} W_{\nu^{\prime \prime}} \operatorname{Tr}\left[\hat{A} \rho_{\nu^{\prime \prime}}(t)\right]}{\operatorname{Tr}[\hat{A}] \sum_{\nu^{\prime \prime}} W_{\nu^{\prime}} \operatorname{Tr}\left[\rho_{\nu^{\prime \prime}}\left(t_{\mathrm{f}}\right)\right]}\right\}^{1 / 2}
$$

where $\rho_{\nu^{\prime \prime}}(t)=\mid \psi_{\mathrm{e}}\left(t, \nu^{\prime \prime}\right\rangle\left\langle\psi_{\mathrm{e}}\left(t, \nu^{\prime \prime} \mid\right.\right.$ is the density matrix on the excited state resulting from excitation of the $\nu^{\prime \prime}$ vibronic level of the ground electronic state by the field. The total density matrix $\rho=$ $\sum_{\nu^{\prime \prime}} W_{\nu^{\prime}} \rho_{\nu^{\prime \prime}}$. The achievement can be rewritten in a Wigner phase space representation as

$$
\alpha(t)=\frac{2 \pi \hbar \sum_{\nu^{\prime \prime}} W_{\nu^{\prime \prime}} \iint \mathrm{d} p \mathrm{~d} q A(p, q) \rho_{\nu^{\prime \prime}}(p, q, t)}{\iint \mathrm{d} p \mathrm{~d} q A(p, q) \sum_{\nu^{\prime \prime}} W_{\nu^{\prime \prime}} \iint \mathrm{d} p \mathrm{~d} q \rho_{\nu^{\prime \prime}}\left(p, q, t_{\mathrm{f}}\right)}
$$

In eq 17 , the target $A(p, q)$ in the phase space representation is

$$
A(p, q)=\frac{1}{\pi \hbar} \exp \left\{\frac{1}{2}\left[\frac{(q-\bar{q})^{2}}{w_{q q}}+\frac{(p-\bar{p})^{2}}{w_{p p}}\right]\right\}
$$

and $w_{p p} w_{q q}=\hbar^{2} / 4$. The target in this equation is exactly the same as the target in eq 4, except that it is written in the Wigner representation, rather than the coordinate representation.

Despite the apparent additional complexity of eqs 14-18, the finite temperature calculations are not much more difficult than the pure state case. The optimal field obtained from the $M$ function of eq 14 is used to propagate independently each initial vibrational state $\left|\nu^{\prime \prime}\right\rangle$ in the sum. The Wigner density matrix for each initial state is calculated as

$$
\rho_{\nu^{\prime \prime}}(p, q)=\frac{1}{2 \pi \hbar} \int_{-\infty}^{\infty} \mathrm{d} s \mathrm{e}^{-i p s} \psi^{*}{ }_{\nu^{\prime \prime}}(q-s / 2) \psi_{\nu^{\prime \prime}}(q+s / 2)
$$

and summed with the appropriate weight $W_{v^{\prime \prime}}$ to form $\rho$. The achievement is then calculated using eq 17. Using this procedure, we repeated the reflectron calculation at a temperature of 300 K.

We find at $T=300 \mathrm{~K}$ that several eigenvalues $\lambda$ of the control eigenequation (eq 5) are significantly different from zero. This is in contrast with the $T=0 \mathrm{~K}$ case, in which only one eigenvalue is important. The Wigner representation of the globally optimal field at $T=300 \mathrm{~K}$ is shown in Figure $3 \mathrm{a}$, and the second best optimal field at $T=300 \mathrm{~K}$ is shown in Figure $3 \mathrm{~b}$. The achievements for these two fields are 0.91 and 0.41 , respectively. Also plotted in Figure 3a is the optimal field for the pure $\nu^{\prime \prime}=$ 0 (or $T=0 \mathrm{~K}$ case) and, in Figure $3 \mathrm{~b}$, the optimal field for the pure $\nu^{\prime \prime}=1$ initial state. The achievement for the $\nu^{\prime \prime}=1$ initial state is 0.82 , compared to 0.94 for the pure $\nu^{\prime \prime}=0$ case.

Figure 3a shows that the globally optimal fields for $T=0 \mathrm{~K}$ and $T=300 \mathrm{~K}$ are almost identical. The optimal fields for the 

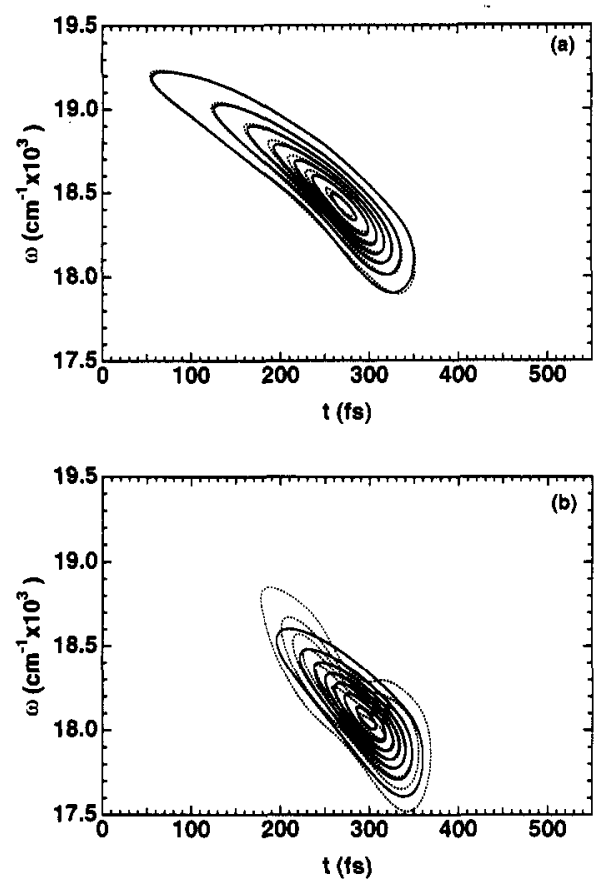

Figure 3. Optimal fields for the reflectron at $T=0 \mathrm{~K}$ and $T=300 \mathrm{~K}$. Globally optimal fields for (a) $T=0 \mathrm{~K}$ (solid contours) and $T=300$ $\mathrm{K}$ (dotted contours). (b) Globally optimal field (eigenfunction with largest eigenvalue $\lambda$ ) for a pure $\nu^{\prime \prime}=1$ initial state (solid contours) and the second best optimal field (eigenfunction with second largest eigenvalue) for $T=300 \mathrm{~K}$ (dotted contours).

second largest eigenvalue and for the pure $\nu^{\prime \prime}=1$, as shown in Figure $3 b$, are also very similar, and clearly different from the globally optimal $T=0 \mathrm{~K}$ and $T=300 \mathrm{~K}$ fields. The $\nu^{\prime \prime}=1$ optimal field is shifted to somewhat later times and lower frequencies than the $\nu^{\prime \prime}=0$ optimal field. This is partly because of the frequency difference between the $\nu^{\prime \prime}=0$ and $\nu^{\prime \prime}=1$ levels of the ground state but also partly because the Franck-Condon factors between these two states and the excited state are different. In particular, some of the higher energy components of the initial $\nu^{\prime \prime}=1$ wave packet are dissociative and do not contribute to the control. The portion of the initial $\nu^{\prime \prime}=1$ state that contributes most strongly to the control has maximum Franck-Condon overlap at larger internuclear distances than the $\nu^{\prime \prime}=0$ case and so requires less time to reach the target. The match between the $\nu^{\prime \prime}=1$ optimal field and the second best optimal field is not perfect, in part because the eigenvector corresponding to the second largest eigenvalue must have a node, but these two fields are certainly more simialr to each other than to the $\nu^{\prime \prime}=0$ optimal field.

Figure 4a shows the Wigner density matrix $\rho\left(p, q, t_{\mathrm{f}}\right)$ resulting from propagation of the wave packet in the presence of the optimal field at $T=0 \mathrm{~K}$. Figure $4 \mathrm{~b}$ shows $\rho\left(p, q, t_{\mathrm{f}}\right)$ for the $T=300 \mathrm{~K}$ case. Also shown in the figures is the initial distribution on the ground state reflected onto the excited state and the target distribution $A(p, q)$. The $T=0 \mathrm{~K}$ case clearly has a better overlap with the target, though the $T=300 \mathrm{~K}$ overlap is still large. The higher temperature wave packet is considerably broader than the $T=0 \mathrm{~K}$ case and does not overlap the target as well in the lower and higher momentum regions of the target. This is reasonable because some fraction of the high-energy components excited by the field are actually dissociative and never reach the target. Similarly, some of the low-energy components will not have sufficient time to reflect from the outer turning point and reach the target with the required momentum.

The result that the $T=300 \mathrm{~K}$ optimal field is so similar to the $T=0 \mathrm{~K}$ field optimal field is at first somewhat surprising, especially since the fractional vibrational populations $W_{\nu}^{\prime \prime}$ for $\nu^{\prime \prime}$ $=0$ and $\nu^{\prime \prime}=1$ at $300 \mathrm{~K}$ are 0.64 and 0.23 , respectively. Two factors conspire to cause this behavior. First, as we discussed in
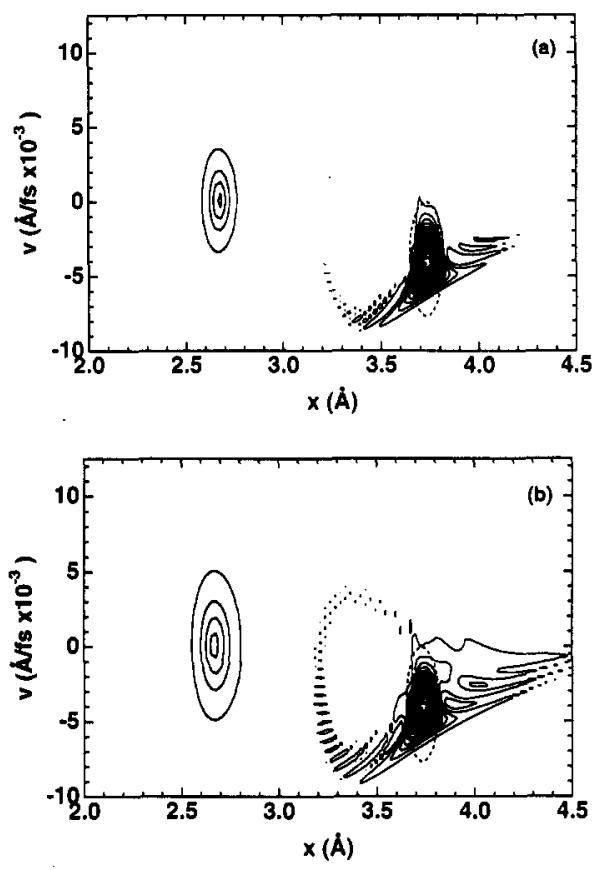

Figure 4. Wigner wave packets $\rho\left(v, x, t_{f}\right)$, where $x$ is the postion and $v$ is the velocity, created by the globally optimal field at (a) $T=0 \mathrm{~K}$ and (b) $T=300 \mathrm{~K}$. Also shown are contours of the initial density (solid contours centered at $2.67 \AA$ ) and the target $A(x, v)$ (dotted contours). The wave packet is plotted as a function of the velocity rather than the momentum for illustrative purposes.

section III, the achievement is very sensitive to the detuning of the excitation frequency. The vibrational constant in $\mathrm{I}_{2}$ is about $214 \mathrm{~cm}^{-1}$, and as shown in Table II, a detuning of $50 \mathrm{~cm}^{-1}$ is enough to decrease the achievement considerably. Second, we are operating in the weak field limit, or linear regime, so the vibrational states cannot mix via a nonlinear mechanism such as Raman scattering. Thus it is not possible, for example, for populations beginning in the $\nu^{\prime \prime}=0$ level to be transferred to $\nu^{\prime \prime}$ $=1$ and then excited by the field to the excited potential surface. In the case of finite temperature, then, the optimization procedure has discovered a somewhat non-intuitive result. Rather than choosing a frequency and chirp that is intermediate between that of the optimal fields for the individual vibrational states, the control equations predict an optimal field very close to the $v^{\prime \prime}=$ 0 result, at the expense of exciting very little of the $\nu^{\prime \prime}=1$ or higher vibrational states. The net result of the finite temperature is a decrease in the achievement, with very little modification to the $T=0 \mathrm{~K}$ optimal field. This again is an indication of the robustness of our results.

\section{Experimental Aspects}

The experimental challenges to realizing practical control over chemical reaction dynamics are considerable. We have emphasized in this paper control of dynamics at the level of the first interaction with light to create a wave packet in a desired region of phase space. This process will in general be followed by a second interaction with light to achieve control over the final fate of the molecule (for example its reaction products). There are experimental difficulties to be overcome associated with both the synthesis of the femtosecond light fields necessary for controlling the dynamics, as well as the coherent detection of the resulting wave packet motion. Fortunately, considerable progress has been made recently in producing the shorter and sufficiently energetic pulses necessary for easier implementation of the first interaction. ${ }^{23}$

On the detection side, wave packet dynamics have been observed with femtosecond temporal resolution by a variety of methods including laser-induced fluorescence from higher lying molecular potential energy surfaces, ${ }^{24,25}$ and by time-resolved stimulated- 


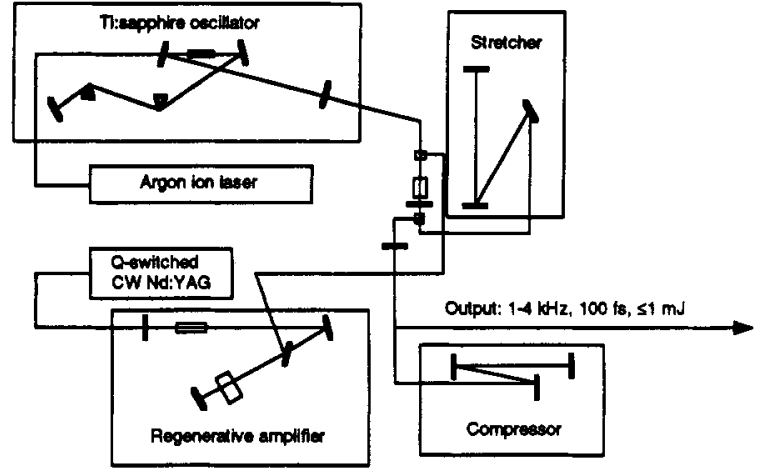

Figure 5. Schematic diagram of amplified femtosecond titanium sapphire (Ti:S) laser system.

emission spectroscopy, in which an initially prepared wave packet on an excited-state surface is stimulated back down to the ground state. ${ }^{26,27}$ Recently, Dunn et al..$^{28}$ have observed the motion of $\mathrm{Na}_{2}$ wave packets by detecting the time-resolved spontaneous emission. For both the preparation and detection of quantum dynamics in gas-phase sysetms, it is advantageous to have available pulses which are as short as possible. We focus in this section on synthesizing the control or preparation light fields. However, the same repertoire of techniques will be needed for the generation of probe fields. Ultrafast diffraction using short X-ray or electron pulses could also, in principle, provide invaluable information on detection of the dynamics, ${ }^{2,29-31}$ and we are working on ways to produce such pulses as well.

As discussed above, we require light fields which have controllable linear and nonlinear frequency chirps and which can be produced at a desired center frequency. Our strategy is to begin with the shortest duration femtosecond pulses which we can make reproducibly, followed by, if necessary, nonlinear optical techniques for shifting the frequency of the pulses to the desired spectral region. Linear filtering can be used to produce the phase and amplitude structure of the optimal light field. We now discuss briefly femtosecond pulse generation, amplification, frequency conversion, and pulse shaping with reference to the experimental apparatus we are constructing.

We are using an amplified femtosecond titanium sapphire (Ti:S) laser system shown schematically in Figure 5. This system is based on the kilohertz chirped pulse amplifier of Salin et al.,23 which is now commercially available from Clark-MXR, Inc. A Ti:S oscillator (Clark Instruments, Inc., Model NJA-4) produces approximately 30 -fs pulses which are then stretched by using an all reflective pulse stretcher to $200-250$ ps. The stretched pulses are seeded into a regenerative Ti:S amplifier pumped by an intracavity doubled Nd:YAG laser (Clark-MXR, Model ORC1000). The high average power of this laser (as much as $40 \mathrm{~W}$ at $532 \mathrm{~nm}$ at a repetition rate of $5 \mathrm{KHz}$ ), coupled with its excellent energy stability $( \pm 1 \%)$, makes this an excellent pump laser. The laser cavity for regenerative amplification uses a wide bandwidth thin film polarizer (ARO, Inc.) which permits limited tuning of the amplified pulse frequency. Amplified pulses with as much as $4.2-\mathrm{W}$ average power have been produced by this laser system at $2.5 \mathrm{kHz}$ with a $40 \%$ slope efficiency. Following recompression with two 1200 lines/mm gratings (with a throughput of $70 \%$ ) the amplified pulse duration is currently approximately 100 fs at 2.5-W average power.

Ti:S oscillators currently produce the shortest pulses of any femtosecond oscillator, and it is now possible ${ }^{32}$ to obtain pulses of less than $20 \mathrm{fs}$. Shorter amplified pulses should be available soon, once aberrations in the pulse stretcher/compressor combination are better compensated. Recently, amplified pulses as short as $40 \mathrm{fs}$ have been obtained. ${ }^{33}$ Thus, we hope to soon have available sub-30-fs pulses with millijoule energies from the Ti:S chirped pulse amplifier.
While tunability in $\mathrm{Ti}: \mathrm{S}$ regenerative amplifiers is possible from approximately $750-850 \mathrm{~nm}$, to produce light at other wavelengths we rely on nonlinear optical techniques. Second, third and fourth harmonic generation can be accomplished using nonlinear optical crystals. In our laboratory, we have achieved up to $50 \%$ conversion efficiency of the infrared into the second harmonic by using type I phase matching in BBO or LBO crystals and up to $6 \%$ total conversion efficiency (or $10 \%$, if one excludes reflection from uncoated optics) into the third harmonic by type I wave mixing in BBO. At the same time, we are able to preserve the femtosecond duration of the laser pulses. Femtosecond pulses in the UV such as these will be a possible source for producing ultrashort electron or X-ray pulses.

Light in spectral regions not covered by harmonics of the Ti:S tuning curve can be produced by white light generation ${ }^{34}$ or by optical parametric generation. ${ }^{35}$ By focusing our millijoule pulses into fused silica substrates of varying thickness, we have been able to produce an extremely broad continuum spanning the ultraviolet to the infrared. Competing optical nonlinearities such as self-focusing can limit the quality of the white light pulses, but if the intensity of the fundamental is not too far above the white light threshold, excellent spatial mode quality and phase coherence are obtainable. Compression of white light pulses down to $16 \mathrm{fs}$ has now been demonstrated. ${ }^{36}$ We have also generated a white light continuum in fused silica using $390-\mathrm{nm}$ second light from our laser. Due to the high pulse energy available with this system, we have chosen to use approximately $3 \%$ of our amplified pulse to generate a continuum. After frequency doubling, we use the remaining light to pump a dye amplifier and amplify a portion of the continuum.

Finally, to realize the optical fields predicted by theory, it is necessary to control both the optical phase and amplitude. The calculations presented earlier describe pulses in which the linear frequency chirp is the prominent feature. Such pulses can be created by passing a transform limited pulse with sufficient bandwidth through a linear dispersive system such as a grating or prism pair. By adding proper lengths of bulk dispersive materials, it is possible to control higher phase terms in the pulse as well. Programmable means for synthesizing femtosecond pulses have also been demonstrated by using liquid crystal modulators to perform spectral filtering. ${ }^{21}$ Using amplitude masking in the pulse stretcher of a chirped pulse amplifier, we have demonstrated control of pulse characteristics at the output of the compressor.

\section{Conclusions}

In this paper, we present an analysis of some of the factors relevant to the experimental observation of weak field control of molecular dynamics. Using the $I_{2}$ molecular reflectron as an example, we show that the globally optimal weak field is simple and robust and can be well fit by a Gaussian functional form with parameters that we believe can be attained in the laboratory. The best fit to the globally optimal field achieves nearly the same degree of control as the globally optimal field. Varying the parameters of the best fit field does reduce the control, but the optimal field is quite robust, even to fairly significant changes in the parameters.

We also showed that thermal vibrational excitation of the ground electron state results in slightly diminished control, but that the globally optimal field for $I_{2}$ near room temperature has an achievement of $87 \%$ of the $T=0 \mathrm{~K}$ case. Interestingly, the optimal fields for $T=0 \mathrm{~K}$ and $T=300 \mathrm{~K}$ are nearly the same. The optimal solution, at least at $300 \mathrm{~K}$ in the weak field limit, is to control the $\nu^{\prime \prime}=0$ portion of the ground-state distribution as well as possible, rather than attempting to use a weighted mean of the optimal fields for the individual vibrational states to control the entire ensemble.

The results of the theoretical calculations have inspired us to attempt the reflectron experiment in the laboratory. We describe 
the apparatus that we are constructing to create and shape the required light fields. The experiment is challenging because it requires intense, short pulses with specified linear and quadratic chirps. Detection of the focused packet adds an additional degree of complexity to the experiment.

Detection is possible by dumping to the ground state in a stimulated-emission pumping (SEP) type experiment, or pumping to a higher lying excited state, in a pump-probe type experiment. The optimal probe field can also be calculated as a control problem in which the pump and probe fieids are optimized sequentially or coherently to maximize an observable on the probe surface. The choice of this observable, as well as the required form of the probe field are important experimental considerations.

Finally, we note that our calculations can be extended by a classical mechanical implementation of control theory. Preliminary results for the reflectron show, at least in the cases studied, that the classical results agree reasonably well with the quantum results. ${ }^{37}$ The classical implementation is more efficient computationally and allows, for example, rotation to be included in the dynamics, and thus in the calculation of the $M$ function and the globally optimal light fields. In addition, the classical theory is more practical for studying large polyatomic molecules, where control of chemically distinct products can be realized, and in treating clusters, surfaces, and condensed phases. It will not be appropriate when interference or tunneling is the dominant process.

Acknowledgment. The calculations in this paper were performed on the Cray Y-MP at the San Diego Supercomputer Center.

\section{References and Notes}

(1) Yan, Y. J.; Gillilan, R. E.; Whitnell, R. M.; Wilson, K. R.; Mukamel, S. J. Phys. Chem. 1993, 97, 2320.

(2) Krause, J. L.; Whitnell, R. M.; Wilson, K. R.; Yan, Y. J.; Mukamel, S. J. Chem. Phys., in press.

(3) Brumer, P.; Shapiro, M. Annu. Rev. Phys. Chem. 1992, 43, 257.

(4) Rabitz, H.; Shi, S. Adv. Mol. Vib. Coll. Dyn. 1991, IA, 187.

(5) Warren, W. S.; Silver, M. S. Adv. Magn. Reson. 1988, 12, 247.

(6) Rice, S. A. Science 1992, $258,412$.

(7) Warren, W. S.; Rabitz, H.; Dahleh, M. Science 1993, 259, 1581.
(8) Yan, Y. J.; Kohler, B. E.; Gillilan, R. E.; Whitnell, R. M.; Wilson,

K. R.; Mukamel, S. Ultrafast Phenomena VIII; Springer: Berlin, 1992.

(9) Tannor, D. J.; Rice, S. A. J. Chem. Phys. 1985, 83, 5013.

(10) Tannor, D. J.; Kosloff, R.; Rice, S. A. J. Chem. Phys, 1986, 85, 5805.

(11) Kosloff, R.; Rice, S. A.; Gaspard, P.; Tersigni, S.; Tannor, D. J. Chem. Phys. 1989, 139, 201.

(12) Somloi, J.; Tannor, D. J. Submitted for publication.

(13) Somloi, J.; Kazakov, V. A.; Tannor, D. J. Chem. Phys. 1993, 85, 172.

(14) Shapiro, M.; Brumer, P. Chem. Phys. Lett. 1993, 208, 193.

(15) Tal-Ezer, H.; Kosloff, R. J. Chem. Phys. 1984, 81, 3967. 483.

(16) Lill, J. V.; Parker, G. A.; Light, J. C. Chem. Phys. Lett. 1982, 89, (17) 1400.

(18) Yan, Y. J.; Mukamel, S. J. Chem. Phys. 1988, 88, 5735.

(19) Heller, E. J. Acc. Chem. Res. 1981, 14, 368.

(20) Averbukh, I.; Shapiro, M. Phys. Rev. A. 1993, 47, 5086.

(21) Weiner, A. M.; Heritage, J. P. Rev. Phys. Appl. 1987, 22, 1619.

(22) Press, W. H.; Flannery, B. P.; Teukolsky, S. A. Vetterling, W. T. Numerical Recipes: The Art of Scientific Computing; Cambridge University Press: Cambridge, U.K., 1986.

(23) Salin, F.; Squier, J.; Mourou, G.; Vaillancourt, G. Opt. Lett. 1991, 16, 1964.

(24) Khundkar, L. R.; Zewail, A. H. Annu. Rev. Phys. Chem. 1990, 41 ,

(25) Bowman, R. M.; Dantus, M.; Zewail, A. H. Chem. Phys. Lett. 1989. $161,297$.

(26) Dai, H. L., Ed. Special Issue on Molecular Spectroscopy and Dynamics by Stimulated-emission Pumping. J. Opt. Soc. Am. 1990, B7 (No. 9).

(27) Chen, Y.; Hunziker, L.; Ludowise, P.; Morgon, M. J. Chem. Phys. 1992, 97, 2149.

(28) Dunn, T. J.; Sweetser, J. N.; Walmsley, I. A.; Radzewicz, C. Phys. Rev. Lett. 1993, 70, 3388.

(29) Bergsma, J. P.; Coladonato, M. H.; Edelsten, P. M.; Kahn, J. D.; Wilson, K. R.; Fredkin, D. R. J. Chem. Phys. 1986, 84, 6151.

(30) van Wonterghem, H.; Rentzeipis, P. M. Appl. Phys. Lett. 1990, 56, 1005 .

(31) Williamson, J. C.; Dantus, M.; Kim, S. B.; Zewail, A. H. Chem Phys. Lett. 1992, 196, 529 .

(32) Huang, C.; Asaki, M.; Backus, S.; Murnane, M.; Kapteyn, H. Nathel,

H. Opt. Lett. 1992, 17, 1289.

(33) Rudd, J. V.; Korn, G.; Kane, S.; Squier, J.; Mourou, G.; Bado, P. Unpublished result.

(34) Alfano, R. R.; Shapiro, S. L. Phys. Rev. Lett. 1970, 24, 592.

(35) Ellington, R. J.; Tang, C. L. Opt. Lett. 1993, 18, 438.

(36) Raksi, F.; Collings, D.; Selvaraj, K.; Schumacher, K. L.; Samuel, I. W. D.; Friend, R. H. Unpublished result.

(37) Krause, J. L.; Whitnell, R. M.; Wilson, K. R.; Yan, Y. J. In Ultrafast Reaction Dynamics and Solvent Effects; P. Rossky, Y, Gauduel, Eds., in press. 